\title{
Journal of

\section{A novel method for the preparation of non-agglomerated nanometre sized particles of lanthanum phosphate phosphors utilising a high surface area support in the firing process}

\author{
Alireza Salimian, George Robert Fern, ${ }^{*}$ Robert Withnall and Jack Silver* \\ Received 18th June 2012, Accepted 31st August 2012 \\ DOI: $10.1039 / \mathrm{c} 2 \mathrm{jm} 33931 \mathrm{e}$

\begin{abstract}
A convenient method is described that uses a quartz wool substrate to immobilise nanometre sized phosphor precursor particles enabling them to be fired at high temperature without sintering/ agglomeration. The nanometre sized phosphor particles are easily removed from the substrate by re-dispersion into liquid for subsequent use.
\end{abstract}

\section{Introduction}

The study of nanometre sized materials is one of the areas of research attracting a great deal of interest amongst material scientists, chemists, physicists, biomaterial scientists, and indeed biologists as well as scientists working in various areas of environmental safety. In the field of molecular tagging of biological molecules a number of nanometre sized particles of light emitting phosphors have been studied. ${ }^{1}$

Lanthanum phosphate (LAP) phosphors have been intensely studied because of their diverse applications in backlit LCD displays, ${ }^{2}$ emissive displays such as plasma display panels and field emission displays, ${ }^{3,4}$ general lighting, ${ }^{5}$ luminescent markers for imaging, ${ }^{6}$ and bio-imaging. ${ }^{1}$ LAP has been demonstrated to be a suitable host lattice for rare earth elements due to its stability, high melting temperature ${ }^{7}$ and efficient light output under $254 \mathrm{~nm}$ excitation when doped with cerium and terbium co-activators. ${ }^{8}$ Various methods of synthesising LAP doped with a number of elements have been investigated including sol-gel, ${ }^{9-12}$ hydrothermal synthesis, ${ }^{13,14}$ wet chemical precipitation, ${ }^{15,16}$ synthesis via sonication, ${ }^{17}$ using microemulsion/polymer. ${ }^{18}$ An interesting hydrothermal synthesis route has been demonstrated as an effective approach for the preparation of nanocrystalline LAP where either rhabdophane or monazite crystalline phases of LAP are produced depending on the temperature of synthesis. ${ }^{19-21}$ However to produce LAP with a narrow particle size distribution by hydrothermal synthesis, complexing agents have to be introduced during the synthesis which subsequently need removing. ${ }^{22}$

Synthesis by ultrasonication proceeds via the formation and collapse of bubbles, referred to as acoustic cavitation, enabling the formation of nanoparticles in the hot spots formed.

Centre for Phosphor and Display Materials, Wolfson Centre for Materials processing, Brunel University, Uxbridge, Middlesex UB8 3PH, UK. E-mail: jack.silver@brunel.ac.uk; george.fern@brunel.ac.uk; Fax: +44 (0)1895 269737; Tel: +44 (0)1895266116
Temperatures of $c a .4700{ }^{\circ} \mathrm{C}$ and pressures of $c a .1800 \mathrm{~atm}$ reached in these hot spots $^{23,24}$ create a suitable nanometre sized environment for the formation of the particles. However the rapid cooling rate associated with this technique hinders crystallisation of the final product and the nanometre sized materials produced are amorphous. ${ }^{25}$ It has been reported that the crystallinity can be improved by using non-volatile compounds for the synthesis, thereby enabling the reaction to occur at closer proximity to hot spots where both amorphous and crystalline nanomaterials are produced. ${ }^{16,26}$ During the synthesis of nanophosphors it is well known that the light emission can be enhanced by a subsequent annealing process. However this will lead to sintering unless the particles are kept apart during this process, consequently nano-phosphors have been bound inside a silica matrix which must subsequently be removed by $\mathrm{HF}$ etching. ${ }^{27}$

In this work, we report a novel alternative route for the synthesis of nanometre sized crystalline LAP:Tb,Ce. This method was based on an earlier report of the synthesis of nanometre sized crystals of $\mathrm{LaPO}_{4}: \mathrm{Eu}$ and $\mathrm{CePO}_{4}: \mathrm{Tb}$ with a mean particle size of $5 \mathrm{~nm}$ and a narrow size distribution. ${ }^{28}$ Our new route utilises part of the previously published synthetic procedure, ${ }^{28}$ whereby the nanometre sized materials are synthesized within an organic matrix comprising of stearic and citric acids. Our modification, reported herein, involves the calcination of the organic-inorganic mixture of materials on a quartz wool substrate.

\section{Experimental}

Samples of lanthanum phosphate doped with terbium and cerium were prepared in an organic matrix by dissolving 0.0333 moles of lanthanum nitrate $(99.99 \%$, Alfa Aesar), 0.0037 moles of terbium chloride ( $99.99 \%$, Aldrich), 0.0024 moles of cerium nitrate $(99.99 \%$, Aldrich) and 0.033 moles of citric acid $(99.5 \%$, Alfa Aesar) in $50 \mathrm{~mL}$ of methanol followed by the addition of 0.1 moles of stearic acid (Fisher). This solution was then heated 
under reflux while stirring for 2 hours. Then $2 \mathrm{~mL}$ of phosphoric acid were added to the solution and allowed to react for 2 hours. The same procedure was repeated, however instead of using phosphoric acid, $3 \mathrm{~mL}$ of a $5 \mathrm{M}$ solution of ammonium phosphate $(99.9 \%$ Fluka) was used. These two sample batches were subsequently named GP and GA, respectively. The hot solution was then gently poured over $20 \mathrm{~g}$ of quartz wool, QW (which had been pre-treated on its own in a furnace at approximately 1000 ${ }^{\circ} \mathrm{C}$ ), on a Buchner funnel so that the wool was covered uniformly by the solution and it was then allowed to cool. The wool was dried under vacuum and $8 \mathrm{~g}$ from each QW sample (GP \& GA) was fired at $1050{ }^{\circ} \mathrm{C}$ for 10 minutes in air. Higher firing temperatures than $1050{ }^{\circ} \mathrm{C}$ and longer firing times than 10 minutes gave stronger luminescence but larger particles, and conversely lower firing temperatures and shorter firing times gave weaker luminescence and smaller particles. The $\mathrm{Ce}$ and $\mathrm{Tb}$ precursor concentrations do not significantly affect the quality of the nanoparticles, although they do influence the PL intensity, as is well known for these co-activators.

Each QW sample was then placed in $300 \mathrm{~mL}$ of iso-propanol followed by sonication and stirring for 30 minutes to disperse the particles. The QW was subsequently removed and the solution was centrifuged at $3000 \mathrm{rpm}$ to remove any residual pieces of QW.

Each iso-propanol solution was used for electrodeposition of LAP nanoparticles onto aluminium stubs for subsequent CL emission analysis at accelerating voltages of $1-5 \mathrm{kV}$. The CL spectra were collected with a TEL 301 Photometer Telescope, with an aperture mirror viewing system to focus precisely on the area of measurement. The photometer telescope collects the light output through the front viewing port of the low voltage chamber. The collected light passes to the M3000 monochromator through the integral flexible fibre optic bundle. These results were compared to data obtained using micrometre sized commercial green-emitting LAP phosphor from Nichia. Using $2 \mathrm{~mL}$ specimens from each sample batch the photoluminescent properties of the material were investigated under $254 \mathrm{~nm}$ excitation using a Bentham Instruments photoluminescent (PL) analyser.

The PL excitation and emission spectra were obtained using a Bentham (Reading, UK) M300 programmable grating monochromator photometer system with computer controlled wavelength scanning and intensity data collection, using in the visible region an 1800 lines per mm grating. The stepping motor and sine drive allows wavelength scanning to be completely controlled from a remote stepping drive unit (SMD3B). The SMD3B in turn is controlled by a PC via its IEEE 488 interface. Sample excitation and collection was collected inside an in-built sealed chamber connected to the M300 monochromator via a fibreoptic bundle.

The crystalline phases of the products were determined by X-ray powder diffraction (XRPD) using a Bruker D8 Advance $\mathrm{X}$-ray powder diffractometer fitted with a nickel-filtered copper source and a LynxEye ${ }^{\mathrm{TM}}$ silicon strip detector. Data were recorded from 5 to $1002 \theta$ degrees at $293 \mathrm{~K}$. The diffractometer was previously calibrated using an aluminium oxide line position standard from Bruker and $\mathrm{LaB}_{6}$ NIST SRM 660a line profile standard. The emission of the nickel filtered $\mathrm{Cu}$ source and hence the instrumental line broadening was determined by fitting the

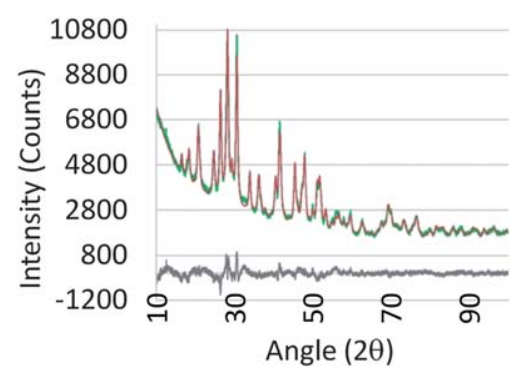

Fig. 1 XRD pattern of the LAP particles and associated crystal parameters for GA sample. Average coherent scattering domain size $=18.1 \mathrm{~nm}$, $a=6.8181 \AA, b=7.0521 \AA, c=6.4910 \AA, \beta=103.42^{\circ}$. Red line $=$ fit, green line $=$ raw data, grey line $=$ difference line.

NIST standard using Bruker Topas version 3 software. The analysis was conducted at ambient temperatures and the samples were prepared as powders (dried powders extracted from the QW) and rotated during the collection of the data to maximise the randomisation. Phases were identified from the XRPD diffractograms by peak search matching using the ICSD PDF-2 data files and CIF files retrieved using the Chemical Database Service. ${ }^{29}$ Rietveld refinement of the diffractograms was carried out to determine the lattice parameters and crystallite sizes. The identifiable phases were refined using Bruker Topas version 3 software.

The morphological properties and particle dimensions of the nanomaterial products were investigated by scanning electron microscopy (Zeiss, Supra 35VP) and transmission electron microscopy (Jeol 2000FX).

\section{Results and discussion}

The particles were characterised by XRPD. The data fits to the known pattern of the pure Monazite phase of LAP (Fig. 1) (PDF number 83-0651) and no other phases are present. The determination of lattice parameters and crystallite sizes by standard Rietveld refinement indicate (Fig. 1) an average coherent scattering domain size of $18.1 \mathrm{~nm}$ which is consistent with the average particle size seen in the TEM study.

Particle sizes and morphologies of the as synthesized LAP:Tb,Ce particles were analysed by scanning (SEM) and transmission electron microscopy (TEM). The particle size and morphology were both independent of the phosphate source used (phosphoric acid or ammonium phosphate). Nanoparticles with a size distribution within the range $11-41 \mathrm{~nm}$ were observed in both GA and GP samples and a histogram for GA is shown in

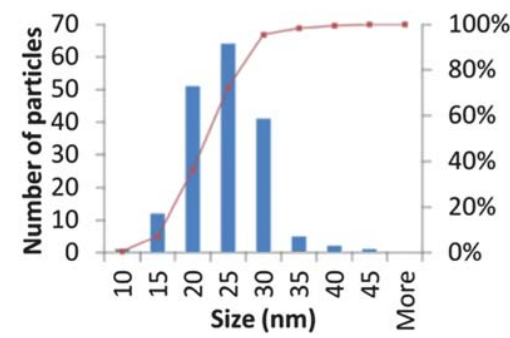

Fig. 2 Histogram of the GA particles demonstrating the narrow size distribution. 

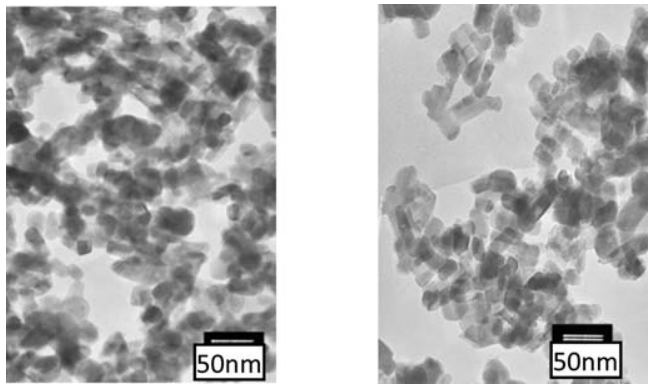

Fig. 3 TEM images of the LAP nanoparticles; (left): phosphoric acid source (GP), (right): ammonium phosphate source (GA). Scale bars = $50 \mathrm{~nm}$.

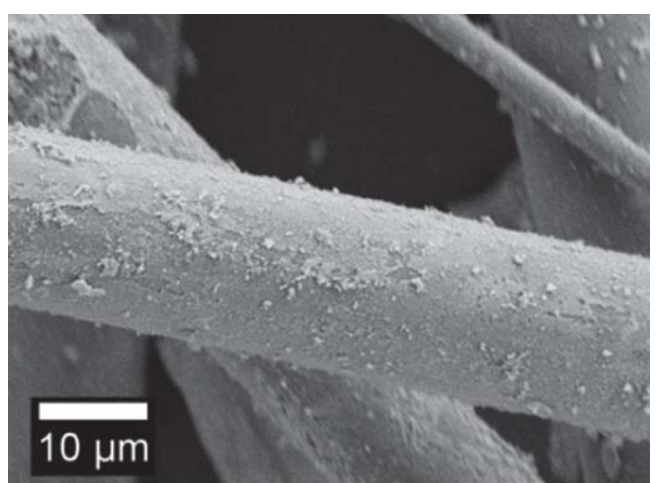

A

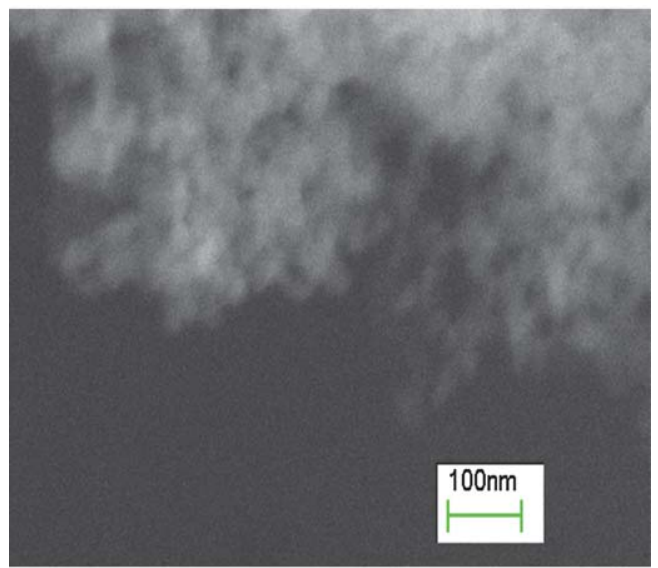

B

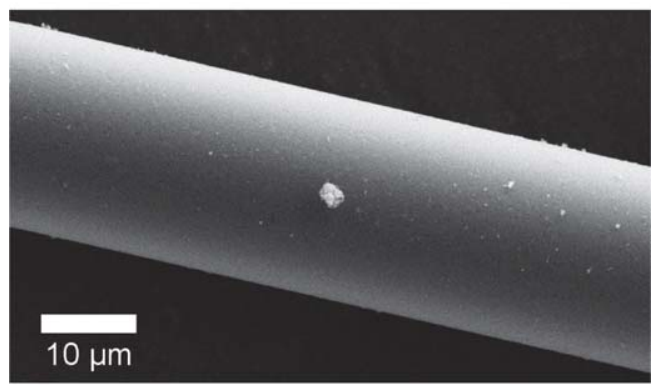

C

Fig. 4 SEM analysis of the quartz wool samples; A (top) and B (middle): QW fibres loaded with the GP sample and fired at $950{ }^{\circ} \mathrm{C}$. C (bottom): untreated QW fired at $950{ }^{\circ} \mathrm{C}$. (Note impurity "particle" is rare in $\mathrm{C}$ compared with the voluminous covering in A and B.) Scale bars $=10 \mu \mathrm{m}$.

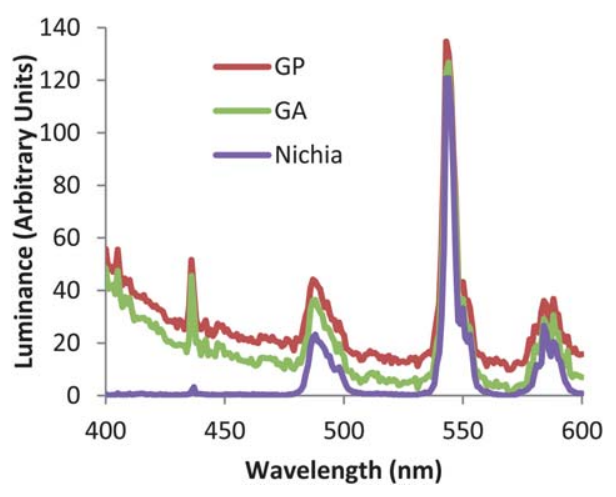

Fig. 5 Photoluminescence spectra of the nanoparticles from each batch dispersed in iso-propanol and excited at $254 \mathrm{~nm}$ compared to the photoluminescence spectrum of a commercial bulk phosphor sample powder. GP (top), GA (middle) and a commercial large particle material (bottom).

Fig. 2. TEM images of the particles are presented in Fig. 3. Whilst some larger crystals are present in Fig. 3, the majority of the particles lie within the lower limit of this range. Fig. 3 demonstrates that pure nano-crystals have been isolated after the combustion firing which has been effective at removing the excess stearic acid used during synthesis and the clarity of the carbon TEM support shows the ease with which the particles can be isolated for further use.

In the SEM micrographs taken of the quartz wool fibres after firing, the formation of a thin layer of LAP on the surface of the fibre is clearly visible (Fig. 4A and B). Higher resolution SEM reveals the particles on the surface (Fig. 4B). An uncoated fibre is presented in Fig. 4C. Note the impurity "particle" is rare in Fig. 4C compared with the voluminous covering in Fig. 4A and $B$. The particles were then separated from the fibre and dispersed in isopropanol by sonication.

The photoluminescent spectra of the materials dispersed in isopropanol (see Fig. 5) clearly manifest an emission peak at $544 \mathrm{~nm}$. Even though the scale for the brightness of the samples is recorded in arbitrary units, visual inspection of the emission of the samples under $254 \mathrm{~nm}$ excitation (see Fig. 6) indicated that the GA sample is the brighter phosphor. PL excitation spectra have previously been reported for LAP:Ce,Tb nanophosphors, and the excitation mechanism has been demonstrated to involve energy transfer between $\mathrm{Ce}^{3+}$ and $\mathrm{Tb}^{3+} .{ }^{30}$

The cathodoluminescent (CL) data were collected from the samples plated onto a finely polished SEM stub. The sample
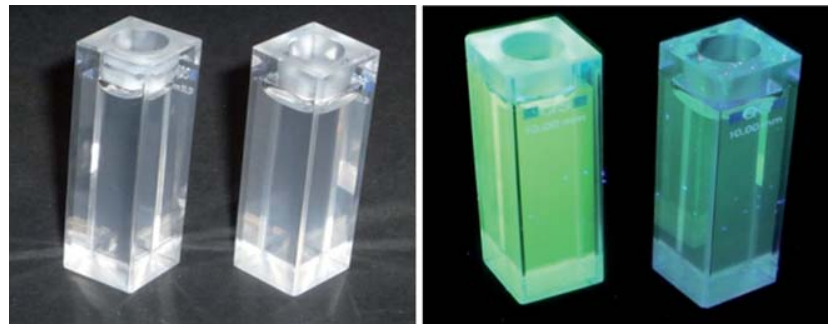

Fig. 6 Photoluminescent emission observation of the nano-particles dispersed in isopropanol under white light (left) and $254 \mathrm{~nm}$ (right) excitation. (GA on the left and GP on the right in each image.) 


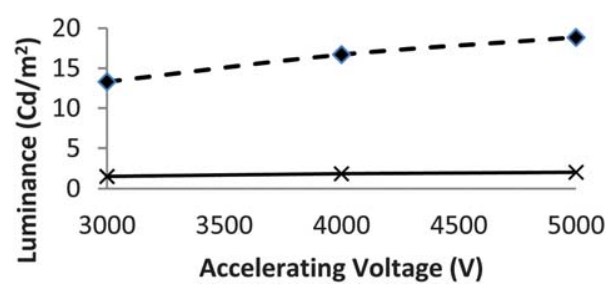

Fig. 7 Cathodoluminescent properties of the nanometre sized phosphor particles. Dashed line is GA, solid line is GP.

coating was a thin layer equivalent to $0.0008 \mathrm{~g}$ of the nanomaterial. The stub was then exposed to an electron beam with a radius that matched that of the stub. The CL emission intensity was then measured at 3,4 and $5 \mathrm{kV}$ from the CL emission spectra, which were in good agreement with those that have previously been reported from LAP:Ce,Tb nanophosphors. ${ }^{30} \mathrm{~A}$ plot of the luminance against the accelerating voltage for the nanometre sized phosphor particles is presented in Fig. 7. Both samples exhibited luminescence but the GA sample was clearly brighter.

\section{Conclusions}

Herein we have demonstrated that:

The quartz wool substrate was a useful material that offers a high surface to volume ratio during the combustion firings of the materials, allowing the nanometre sized particles to crystallise with minimal sintering.

The nanometre sized particles are easily separated from the quartz wool via gentle sonication and can be dispersed easily in any medium for further application.

The nanometre sized particles of lanthanum phosphate were produced in the monazite phase having a particle size distribution in the range of $11-41 \mathrm{~nm}$.

The source of the phosphate ion did not affect the size or crystal morphology of the particles, however it may affect the cathodoluminescent and photoluminescent properties of the particles produced.

This simple technique of using the large surface area of quartz wool as a substrate when firing the nanometre sized particles has been demonstrated herein. The method offers potential for scale up, hence offering an alternative to the hydrothermal and sonication techniques for producing nanometre sized particles, where large scale production can be expensive and complicated.

\section{Acknowledgements}

We wish to thank the BBSRC for grant funding this work (BBF0050161, NanoProbes; Development of novel probes for biological submicroscopic multicolour imaging). We wish to acknowledge the use of the EPSRC funded Chemical Database Service at Daresbury.

\section{Notes and references}

1 K. Byrappa, M. K. Devaraju, J. R. Paramesh and K. Soga, J. Mater. Sci., 2008, 43, 2229.

2 R. Withnall, J. Silver, C. Catherall and G. R. Fern, SID Int. Symp. Dig. Tech. Pap., 2008, 39, 1663.

3 Y. C. Kang, E. J. Kim, D. Y. Lee and H. D. Park, J. Alloys Compd., 2002, 347, 266.

4 R. P. Rao, J. Lumin., 2005, 113, 271.

5 C. Feldmann, T. Justel, C. R. Ronda and P. J. Schmidt, Adv. Funct. Mater., 2003, 13, 511.

6 S. Heer, O. Lehman, M. Hasse and H. U. Gudel, Angew. Chem., Int. Ed., 2003, 42, 3179.

7 S. Lucas, E. Champion, D. Bernache-Assollant and G. Leroy, J. Solid State Chem., 2004, 177, 1312.

8 G. Blasse and B. C. Grabmeier, Luminescent Materials, SpringerVerlag, New York, 1994

9 R. P. Rao, J. Electrochem. Soc., 2003, 150, H165.

10 K. K. Chawala, H. Liu, J. Janczak-Rusch and S. Sambasivan, J. Eur. Ceram. Soc., 2000, 20, 551.

11 M. Yu, H. Wang, C. K. Lin, G. Z. Li and J. Lin, Nanotechnology, 2006, 17, 3245.

12 M. Yu, J. Lin, J. Fu, H. J. Zhang and Y. C. Han, J. Mater. Chem., 2003, 13, 1413.

13 Y. Fujishiro, H. Ito, T. Sato and A. Okuwaki, J. Alloys Compd., 1997, 252, 103.

14 P. Yang, Z. Quan, C. Li, Z. Hou, W. Wang and J. Lin, J. Solid State Chem., 2009, 182, 1045.

15 S. Lucas, E. Champion, D. Bregioux, D. Bernache-Assollant and F. Audubert, J. Solid State Chem., 2004, 177, 1302.

16 R. Kijikowska, J. Mater. Sci., 2003, 38, 229.

17 S. S. Brown, H. Im, A. J. Rondinone and S. Dai, J. Colloid Interface Sci., 2005, 292, 127.

18 R. Chai, H. Lian, P. Yang, Y. Fan, Z. Hou, X. Kang and J. Lin, J. Colloid Interface Sci., 2009, 336, 46.

19 Y. W. Zhang, Z. G. Yan, L. P. You, R. Si and C. H. Yan, Eur. J. Inorg. Chem., 2003, 4099.

20 M. Hasse, K. Riwotski, H. Meysssamy and A. Kornowski, J. Alloys Compd., 2000, 303-304, 191.

21 W. B. Bu, Z. L. Hua, L. X. Zhang, H. R. Chen, W. M. Huang and J. L. Shi, J. Mater. Res., 2004, 19, 2807.

22 V. Buissette, M. Moreau, T. Gacoin, J. P. Boilot, J. Y. Chaneching and T. Le Merceir, Chem. Mater., 2004, 16, 3767.

23 K. S. Suslick, Science, 1990, 247, 1439.

24 E. B. Flint and K. S. Suslick, Science, 1991, 253, 1397.

25 A. Gedanken, Ultrason. Sonochem., 2004, 11, 47.

26 K. S. Suslick, D. A. Hammerton and R. E. Cline, J. Am. Chem. Soc., 1986, 108, 5641.

27 A. Revaux, G. Dantelle, N. George, R. Seshadri, T. Gacoin and J.-P. Boilot, Nanoscale, 2011, 3, 2015.

28 L. N. Ho, H. Nishiguchi, K. Nagaoka and Y. Takita, J. Rare Earths, 2007, 25, 684.

29 D. A. Fletcher, R. F. McMeeking and D. Parkin, The United Kingdom chemical database service, J. Chem. Inf. Comput. Sci., 1996, 36, 746.

30 Z. Hou, L. Wang, H. Lian, R. Chai, C. Zhang, Z. Cheng and J. Lin, J. Solid State Chem., 2009, 182, 698. 